\title{
Two-dimensional gel studies of genetic variation in the plasma proteins of Amerindians and Japanese
}

\author{
Jun-ichi Asakawa ${ }^{1}$, Norio Takahashi ${ }^{1}$, Barnett B. Rosenblum ${ }^{2}$, and James V. Neel ${ }^{2}$ \\ ${ }^{1}$ Division of Biochemical Genetics, Department of Clinical Laboratories, Radiation Effects Research Foundation, Hiroshima 730, Japan \\ ${ }^{2}$ Department of Human Genetics, University of Michigan Medical School, Ann Arbor, MI 48109, USA
}

Summary. Genetic variation has been studied in plasma samples from 107 Amerindian children and their parents, and 110 Japanese children and their parents by means of two-dimensional polyacrylamide gel electrophoresis. Twenty-three polypeptides were scored; the identity of nine of these is at present still unknown. Genetic variation was encountered in 11 of these polypeptides. We have previously reported that the index of heterozygosity was $6.2 \pm 0.7 \%$ for 20 "randomly selected", silver stained polypeptides scored for genetic variation in Caucasoids (Rosenblum et al. 1983b). For technical reasons only 11 of these 20 polypeptides could be routinely scored in preparations from the Amerindian samples. For these 11 polypeptides, the indices of heterozygosity in the three populations were: Amerindians, $4.5 \pm 0.6 \%$; Japanese, $5.7 \pm 0.7 \%$; Caucasoids, $8.0 \pm 1.1 \%$. Even with these relatively small numbers some striking ethnic differences as regards individual polypeptides are apparent.

\section{Introduction}

Recently, employing two-dimensional polyacrylamide gel electrophoresis (2-D PAGE) and a combination of Coomassie Blue staining and the sensitive silver stain developed by Sammons et al. (1981), we have reported on the genetic variation encountered in the polypeptides of plasma samples from a predominantly Caucasoid population (Rosenblum et al. $1983 \mathrm{~b}$ ). For a series of 20 polypeptides best visualized after silver-staining, whose identity was unknown at the time they were selected for study, and which were selected solely because it appeared variants if present could be readily detected, the heterozygosity index was $6.2 \pm 0.7 \%$. For a different series of polypeptides best scored after Coomassie Blue staining, but whose selection for various reasons cannot be regarded as unbiased with reference to variability, the index of heterozygosity was variously $5.1 \pm 0.9 \%$ (Neel et al. 1984) or $8.7 \pm 1.1 \%$ (Rosenblum et al. 1983b), depending on the polypeptides scored. All variants were confirmed as genetic in nature by the occurence of the same variant in one parent or the other. For a series of 46 erythrocyte lysate polypeptides selected without reference to variability, we encountered a heterozygosity index of $3.1 \pm 0.5 \%$ (Rosenblum et al. 1984).

Offprint requests to: Jun-ichi Asakawa, Division of Biochemical Genetics, Department of Clinical Laboratories, Radiation Effects Research Foundation, Hiroshima 730, Japan
These results are in marked contrast to the findings of earlier investigators who, examining human fibroblasts and kidney and brain tissue, reported heterozygosity indices which averaged less than 1\% (McConkey et al. 1979; Walton et al. 1979; Smith et al. 1980; Comings 1982). More recently, several investigators have reported a level of genetic variability in lymphocytes approximately intermediate between the early findings and our own (Hamaguchi et al. 1981, 1982a, b, c; Goldman and Merril 1983). The striking difference in the results of the various studies could be due to three, not mutually exclusive explanations. First, there are differences in the types of preparations under consideration (plasma, erythrocyte lysates, lymphocytes, human brain proteins, human kidney proteins). Second, three different techniques have been used to identify the location of the polypeptides (radioisotope labeling, staining with Coomassie Blue, staining with a silver preparation). Third, we believe we have been more conservative than most investigators in our criteria for a "scorable" polypeptide.

In the present communication we will extend our observations on variation in plasma proteins to two additional mongoloid populations, namely, Amerindians from Central and South America, and Japanese from Hiroshima and Nagasaki. The findings in these two populations will in general confirm the findings on Caucasoids regarding the levels of heterozygosity in plasma proteins although, not unexpectedly, ethnic differences are beginning to emerge. A preliminary report on this material has been published (Asakawa et al. 1984).

\section{Subjects and methods}

\section{Subjects}

At this stage in the evolution of the 2-D PAGE technology, we find it convenient - indeed, indispensable - to prepare the gels in trios derived from a child and his/her father and mother. This not only permits the immediate verification of an apparent genetic variant in a child but, as we will see, reveals the presence of null variants which would otherwise be very difficult to detect. The 107 Amerindian trios examined in this study were drawn from nine tribes, as follows: Yanomama (30); Makiritare (22); Piaroa (12); Macushi (9); Wapishana (10); Guaymi (9); Ticuna (4); Baniwa (10); Cashinawa (1). References to the studies in the course of which the necessary blood samples were obtained will be found in Neel (1978), 
Neel et al. (1980a), and Barrantes et al. (1982). These blood samples had usually been 5-7 days in transit from the field, not always under ideal conditions. In addition, unacculturated, indigenous, tropical-dwelling populations, including Amerindians, characteristically exhibit elevated gamma globulin levels (Neel et al. 1964); the gamma globulins migrate as a diffuse band on 2-D PAGE and, given the amphoteric nature of protein, may influence the positions assumed in the gel by other nearby proteins. As we shall see, these facts introduced difficulties into the scoring of certain polypeptides which had been readily scored in a Caucasian population. The samples had been processed immediately on receipt in the laboratory and aliquots of serum or plasma, as the case may be, have been stored in liquid nitrogen for from 6 to 14 years. In this study, 65 of the Amerindian trios were analyzed on the basis of serum samples and 42 on the basis of plasma samples. In the case of the latter, ACD solution had been the anticoagulant. $\alpha-, \beta-$, and $\gamma$-fibrinogen (see below) could of course not be scored in the preparations from serum samples.

The 110 trios of Japanese blood samples were obtained in the course of the study of the genetic effects of the atomic bombs in Hiroshima and Nagasaki, one aspect of which involved the examination of some 30 proteins by one-dimensional electrophoresis (1-DE) (Neel et al. 1980b), and had been immediately refrigerated and usually processed within $72 \mathrm{~h}$ of collection. ACD solution was used as anticoagulant. Aliquots of the plasma samples used in this study had been stored in liquid nitrogen for from 2 to 24 months. Mother-father-child samples were available in storage because a family study had been conducted in connection with the demonstration of a rare variant in the child (Neel et al. 1980b); this introduced an ascertainment bias with reference to transferrin (see below).

\section{Methodology}

The conditions under which the 2-D PAGE was performed were as described in Neel et al. (1984), with one minor difference; the second dimension was run at $100 \mathrm{~V}$ for approximately $18 \mathrm{~h}$, the exact timing determined by the position of the gel front. Where contrasts with the results of 1-DE are drawn, the methods are referenced in the papers cited.

Choice of polypeptides for scoring. In general, the polypeptides selected for the study of genetic variation were as described in Rosenblum et al. (1983a,b). There were thus two groups of polypeptides. The first set was selected for study from inspection of silver-stained gels, on the basis of certain desirable characteristics: reproducibility, relative isolation on the gel, and a density such that were a variant to occur (with approximately half the normal density), it would be readily recognized. The identity of these polypeptides was unknown at the time to the individual who selected them and insofar as possible, selection for study was without reference to variability. The second set was a group of generally more abundant polypeptides, readily visualized with the Coomassie Blue stain, many of known identity and previously studied with respect to genetic variation, some located in "crowded" portions of the gels. They were also scored for variation but in contrast to the first set, we do not regard them as randomly selected and suitable for inclusion in the data base for an index of heterozygosity. The system of nomenclature for the polypeptides judged as suitable for scoring is identical with that employed previously, being an essentially arbitrary designa- tion based on position on the gel (Neel et al. 1984). To this arbitrary designation is coupled the identity of the polypeptide when known.

Certain of the polypeptides visualized after silver-staining and deemed suitable for scoring in the earlier study could not be reliably scored in the preparations from the Amerindians (A-01, C-08, C-09, D-07, D-08, D-09, D-10, D-11), perhaps for the reasons mentioned earlier (most of these polypeptides migrate adjacent to but at a lower molecular weight than gamma globulin). In addition, the fibrinogens are absent from the Amerindian preparations utilizing serum. We are thus left with 23 polypeptides which can be compared across the three ethnic groups for the occurrence of genetic variants. The positions on the gel of the polypeptides scored in this study are indicated in Fig.1, the nomenclature identical with that employed earlier. Approximate molecular weights of the proteins under consideration are also shown in Fig. 1. Variable numbers from system to system are due to the occurrence of preparations in which a specific polypeptide could not be scored unambiguously, usually because of faintness of staining. Finally, the spot $\mathrm{C}-15$, scored earlier, has now been shown to be a derivative of C-16 (apolipoprotein A-I); to score it separately would be redundant.

\section{Results}

\section{Variable polypeptides}

Phenotype and genotype frequencies for the polypeptides found to be variable in this study or the preceding similar study on Caucasoids are given in Tables 1 and 2. Table 2 also presents the results of tests for agreement with Hardy-Weinberg equilibrium for systems where homozygotes for variants were observed. Although the numbers for any particular system are still relatively small, some noteworthy ethnic differences seem to be emerging, best treated on a system-bysystem basis, first those analyzed after silver staining, then those analyzed after staining with Coomassie Blue.

C-01 (Antithrombin III). No variants of this polypeptide were encountered in either of the two populations, in contrast to the finding of a low-frequency polymorphism in Caucasoids. Kera et al. (1983), employing analytical agarose gel isoelectric focusing followed by immunofixation, encountered one instance each of three different antithrombin III variants in 370 Japanese samples. The studies necessary to determine if these variants would be detected in our system have not been performed.

C-14 (Apolipoprotein E). The ability of 2-D PAGE to detect variants of this system has been demonstrated by Børresen and Berg (1981), Zannis et al. (1981), and Utermann et al. (1982). In Japanese there are both an anodally (Apo E 2) and cathodally (Apo E 4) migrating variant, which show the same mobilities as the correspondingly designated variants in Caucasoids. The Amerindians thus far lack the Apo E 2 variant.

$D-05$. We have observed in both the Amerindian and Japanese samples a second polypeptide about $10 \mathrm{~mm}$ cathodal to the polypeptide originally designated $\mathrm{D}-05$, which varies concurrently with D-05. Both Amerindian and Japanese populations possess an anodally migrating variant of this pair of unidentified polypeptides, of which the anodal one may be identical with that of Caucasians described by Rosenblum et al. 


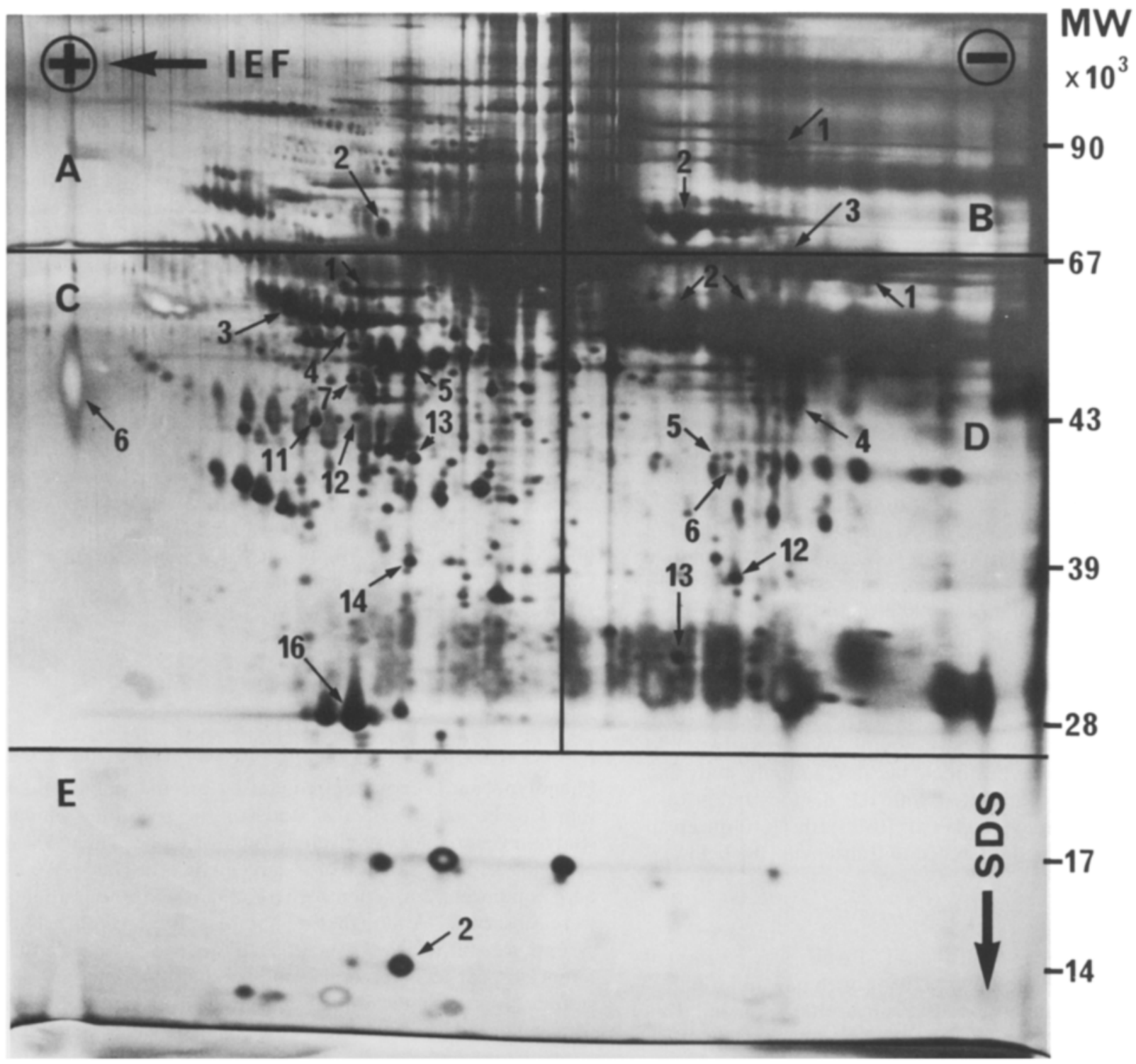

Fig.1. Silver-stained 2-D PAGE pattern of plasma proteins. The gel has been subdivided into five convenient regions and the polypeptides analyzed for this study designated as described by Rosenblum et al. (1983a) and Neel et al. (1984). Tables 1 and 2 provide designations for these proteins where known

(1983b). We designate these variants of D-05 as $V_{1}$ and $V_{1}{ }^{\prime}$. The findings in an individual heterozygous for the variant mentioned above are illustrated in Fig. 2. It is not clear which of these polypeptides is the primary gene product; arbitrarily we will assign this status to the more anodal, designating it $\mathrm{N}$, and the companion polypeptide as $\mathrm{N}^{\prime}$. In general, $\mathrm{N}^{\prime}$ and $\mathrm{V}_{1}{ }^{\prime}$ appear to have slightly greater molecular weights than $\mathrm{N}$ and $\mathrm{V}_{1}$.

In addition, the Japanese possess in polymorphic proportions a previously undescribed variant of $\mathrm{N}$ and $\mathrm{N}^{\prime}$, characterized by altered mobility in both the molecular weight and IEF axes, migrating about $1 \mathrm{~mm}$ above the normal position, as well as about $13 \mathrm{~mm}$ anodally (Fig. 2). In heterozygotes the normal and variant polypeptides have about the same staining intensity. We designate this variant $\mathrm{V}_{2}$ and $\mathrm{V}_{2}^{\prime}$. In the Japanese sample there is a near-significant departure from the HardyWeinberg phenotypic equilibrium proportions expected on the basis of a 3-allele system (Table 2). The reason for this will become apparent later.
A-02 (Hemopexin). Three Japanese exhibited an identical, previously undescribed variant about $1 \mathrm{~mm}$ anodal to the normal position and slightly more intensely staining than the normal polypeptide in a heterozygote (Fig. 3A). Both polypeptides were absent from a gel prepared following treatment of the plasma with an anti-hemopexin antiserum (Dakopatts/ Denmark).

B-02 (Transferrin). The ability of 2-D PAGE to distinguish transferrin variants has been demonstrated by Anderson and Anderson (1977). Nine of the Japanese trios in the total sample of 110 had been assembled for family studies of a transferrin variant encountered in that portion of the study of the genetic effects of the atomic bombs which employs 1-DE. They are excluded from consideration with respect to this system (all nine variants were detected with 2-D PAGE). In the remaining scorable 101 samples, one cathodally migrating and one anodally migrating transferrin variant were detected in Japanese and none in Amerindians. These variants had also 
Table 1. Phenotypic frequencies for the polypeptides scored in all three ethnic groups and found variable in one or more of the groups

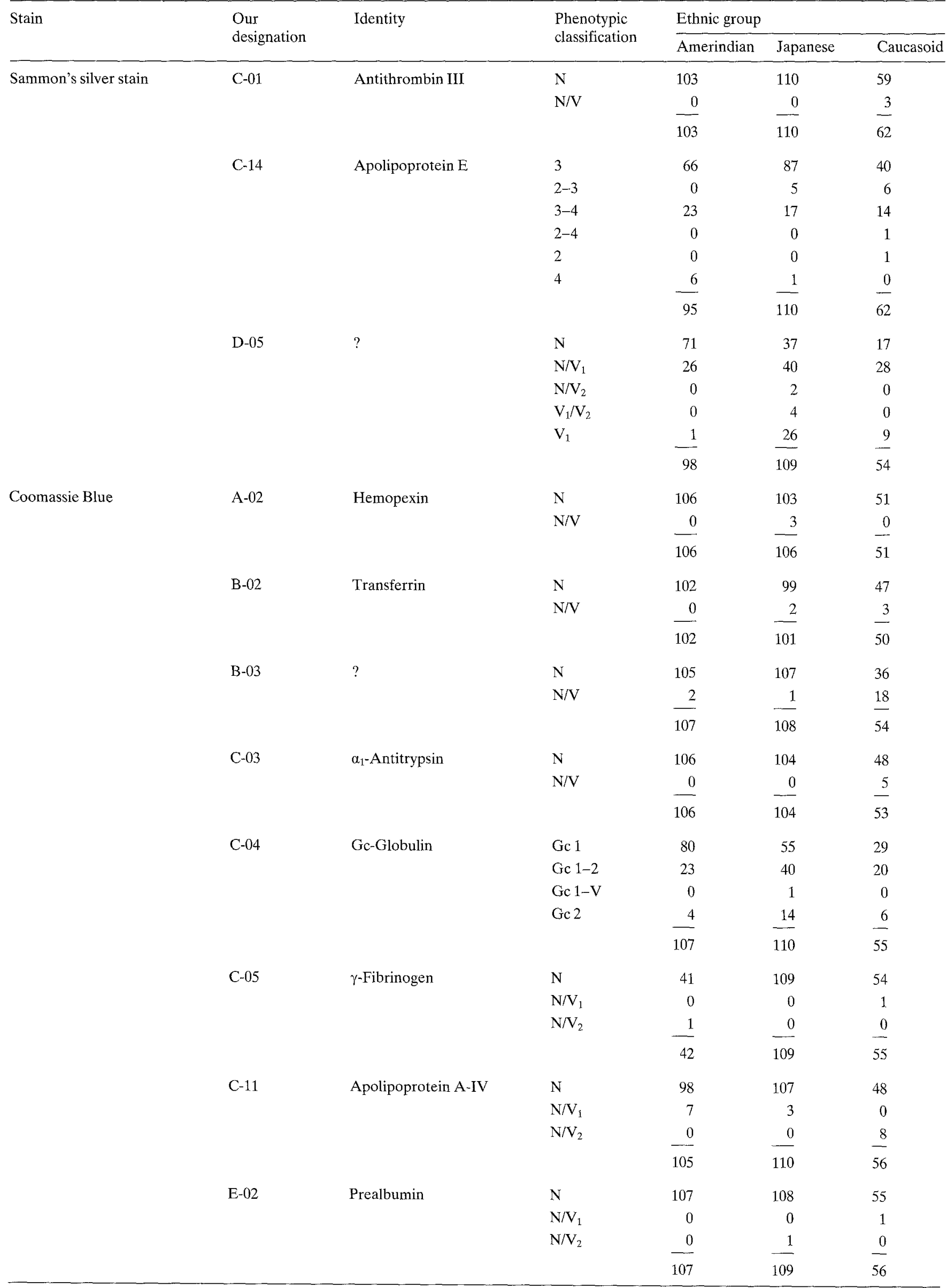


Table 2. Allele frequencies for the variable polypeptides encountered in Amerindians, Japanese, and Caucasians. A $\chi^{2}$ test for agreement with Hardy-Weinberg proportions is performed

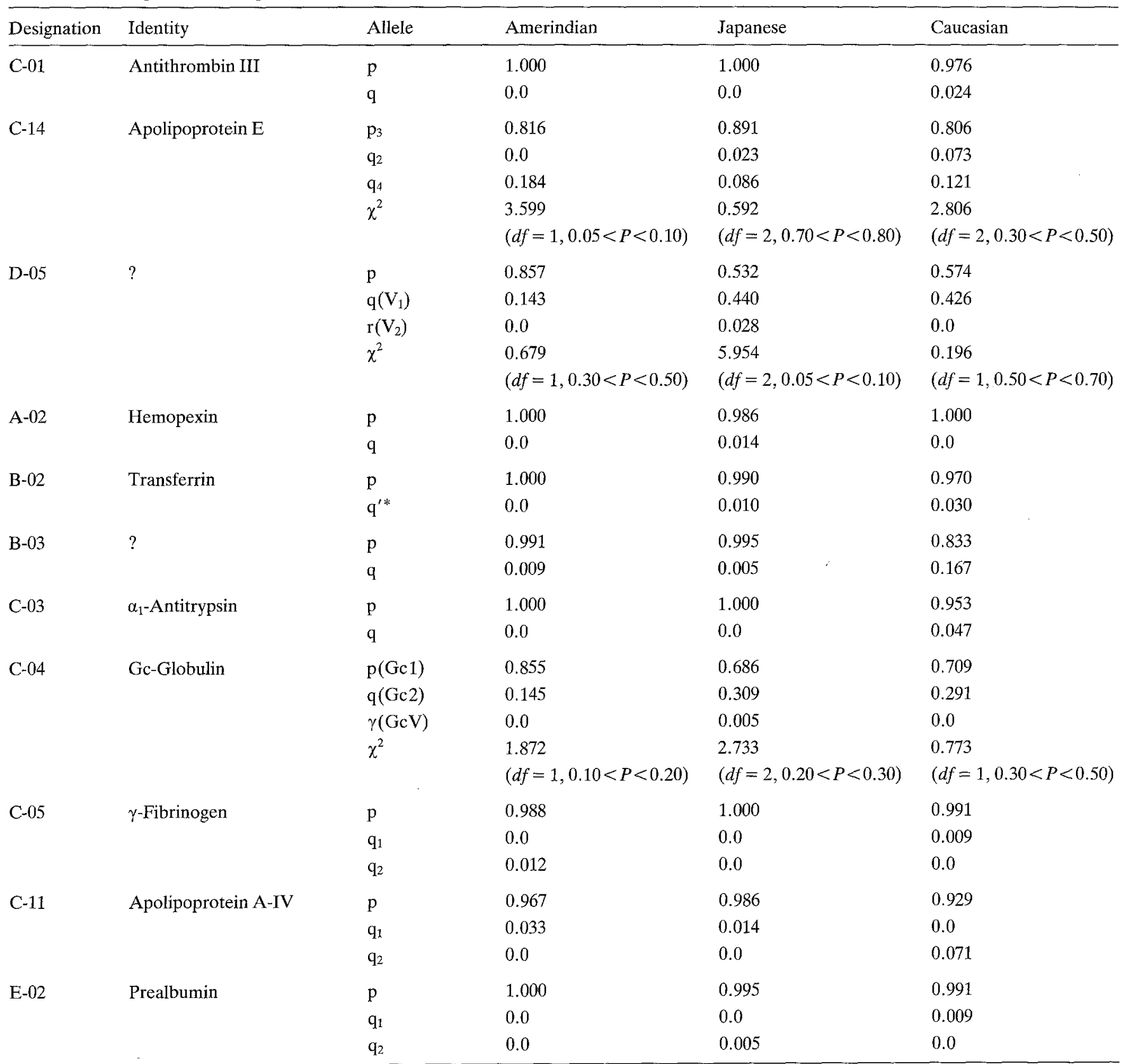

$* \mathrm{q}^{\prime}=$ Several alleles
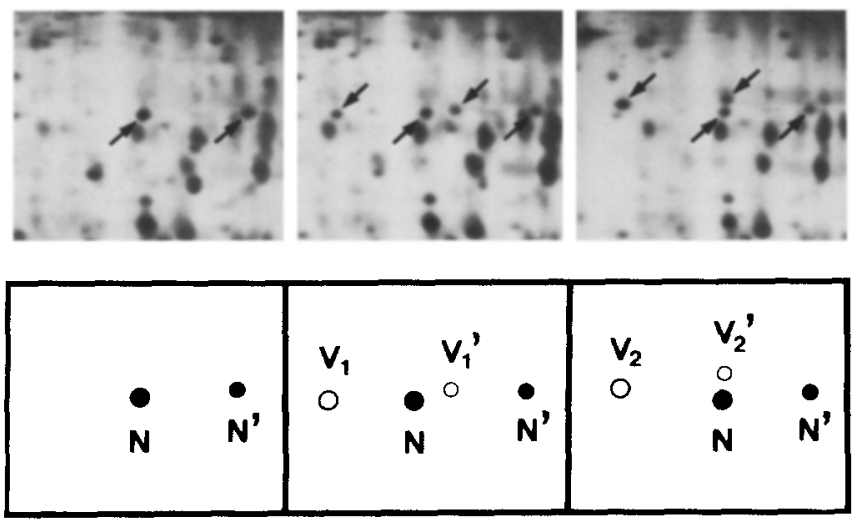

Fig. 2. Photographs and diagrammatic representations of genetic variation involving (unidentified polypeptide) D-05. A detailed description of the findings is in the text been detected by 1-DE in the above referenced study and classified as $\mathrm{Tf} \mathrm{D}_{\text {chi }}$ and $\mathrm{Tf} \mathrm{B}_{\mathrm{HR} 3^{\prime}}$, respectively (Fujita private communication).

$B$-03. A single type of anodally migrating variant was encountered in two Amerindians and one Japanese which was apparently identical to a variant encountered with a much higher frequency in Caucasians (Rosenblum et al. 1983a).

C-03 ( $\alpha_{1}$-Antitrypsin). This polypeptide appeared invariant in the two populations. We had earlier observed in 2-D gels from Caucasoids the polymorphisms for which this systems is well known (Neel et al. 1984), but unlike Brown (1982), we do not feel we can subtype accurately.

C-04 (Gc-globulin). Tracy et al. (1982) demonstrated that 2-D PAGE would distinguish between the common allelic forms of 
A
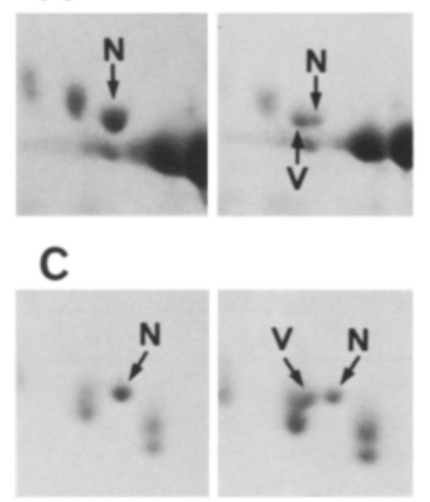

B

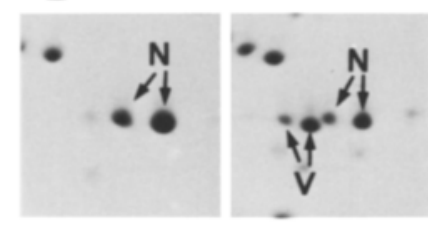

D

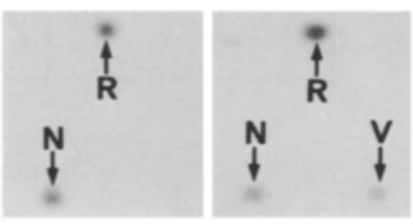

Fig.3A-D. Four new genetic variants of plasma proteins. A A-02, Hemopexin; B C-05, Fibrinogen $\gamma$-chain; C C-11, Apolipoprotein A-IV; D E-02, Prealbumin; N: Normal; V: Variant; R: Reference spot

Mother

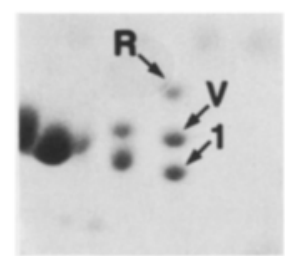

Child
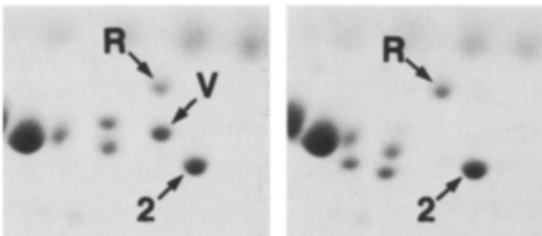

Fig.4. A new genetic variant of Gc-globulin. 1 : $\mathrm{Gc} 1,2$ : Gc 2, $V$ : Gc $\mathrm{V}, R$ : Reference spot

Gc, namely Gc 1 and Gc 2. In this study, these alleles were encountered in both populations, in the proportions reported in the literature (Mourant et al. 1976). Subtypings of the Gc 1 and $\mathrm{Gc} 2$ phenotypes were not attempted. In addition, a single new variant was encountered, of apparently greater molecular weight than Gc 1, in combination with Gc 2 . As Fig. 4 shows, the mother was Gc 1-V, the father type Gc 2, and the child type $\mathrm{Gc} 2-\mathrm{V}$. The variant as well as the polypeptides identified as Gc 1 and Gc 2 were absent from gels prepared following treatment of the plasma with anti-Gc-globulin antiserum (Dakopatts/Denmark).
C-05 ( $\gamma$-Fibrinogen). Variants of this system have been demonstrated with 2-D PAGE by Olaisen et al. (1982) and Rosenblum et al. (1983a). A single previously undescribed variant was encountered in an Amerindian. Gamma-fibrinogen presents on a 2-D gel as a pair of major and a pair of minor spots (Fig. 3B). The variant presented as a pair of major spots anodal to the normal position plus a similar faintly staining pair of minor spots. This variant appears to differ from one described by Olaisen et al. (1982), but our electrophoretic conditions differed slightly from theirs. The identity of our variant as $\gamma$-fibrinogen was established with an anti- $\gamma$-fibrinogen antiserum (Dakopatts/Denmark).

C-11 (Apolipoprotein A-IV). Variation with respect to this polypeptide in 2-D PAGE preparations has been described by Tracy et al. (1982) and Rosenblum et al. (1983a), at a time when the identity of the spot was unknown. We have now identified it as apolipoprotein A-IV through the immunosubtraction technique, employing an anti-apolipoprotein A-IV antiserum kindly provided by Dr. S. Koga of Kyushu University. Both the Amerindian and Japanese samples failed to exhibit the variant encountered in Caucasoids, but were found to have a previously undescribed variant in the IEF axis, migrating anodally about $2 \mathrm{~mm}$ from the normal position (Fig. 3C).

E-02 (Prealbumin). A variant of prealbumin in the molecular axis of a 2-D PAGE gel was recognized by Rosenblum et al. (1983a). A single apparently different and undescribed variant was encountered in a Japanese (Fig. 3D). It migrates about $12 \mathrm{~mm}$ cathodal to the normal position, appears to have a slightly higher molecular weight than the normal polypeptide, and stained slightly less intensely than the normal spot in the heterozygote. Proof of the identity of the variant is provided by its coprecipitation with prealbumin by an antiserum to prealbumin (Dakopatts/Denmark).

\section{Non-variable polypeptides}

The polypeptides found invariant in this study are listed in Table 3 . To our knowledge, only one of these, plasminogen, has been found to exhibit a genetic polymorphism by other investigators (cf. Hobart 1979). It seems very possible 2-D PAGE failed to detect this variant.

Table 3. Systems, and numbers examined, for which all three populations have been invariant to date

\begin{tabular}{|c|c|c|c|c|c|}
\hline Stain & $\begin{array}{l}\text { Our } \\
\text { designation }\end{array}$ & Identity & \multicolumn{3}{|l|}{ Ethnic group } \\
\hline \multirow[t]{5}{*}{ Sammon's silver stain } & $\mathrm{C}-07$ & $?$ & 97 & 110 & 61 \\
\hline & $\mathrm{C}-13$ & $?$ & 102 & 108 & 62 \\
\hline & $\mathrm{C}-16$ & Apolipoprotein A-I & 107 & 110 & 62 \\
\hline & $\mathrm{D}-12$ & $?$ & 99 & 109 & 61 \\
\hline & D-13 & $?$ & 86 & 110 & 55 \\
\hline \multirow[t]{2}{*}{ Coomassie Blue } & B-01 & Plasminogen & 95 & 108 & 47 \\
\hline & $\mathrm{C}-06$ & $\alpha_{1}$ Acid glycoprotein & 106 & 107 & 56 \\
\hline
\end{tabular}




\section{Evidence for the genetic nature of the variants; null alleles in} two of the systems

As noted earlier, gels were run as mother-father-child trios. Each time a child's gel was scored, the gels of the parents were also scored. A total of 254 variants in the homozygous or heterozygous state have been encountered in the subjects of this study. The genetic pattern of codominant inheritance was observed with respect to 251 of these variants. There were, however, three apparently exceptional genetic patterns revealed when the child was compared with its parents, plus six exceptional patterns which became apparent only when the pattern of the parents was compared with that of their child. These findings are distributed over two systems, as follows:

C-04 (Gc-globulin). Three exceptional patterns were encountered in Japanese: two instances of a Gc $1 \times$ Gc 2 mating resulting in a $\mathrm{Gc} 1$ child, and one instance of a Gc $1 \times \mathrm{Gc} 1-2$ mating with a Gc 2 child. The possibility of a discrepancy between legal and biological parentage was investigated with 17 polymorphic genetic systems (ABO, Rh, MNSs, P, Kell, Duffy, haptoglobin, adenosine deaminase, 6-phosphogluconate dehydrogenase, phosphoglucomutase-1, phosphoglucomutase-3, acid phosphatase, esterase D, glutamate oxalate transaminase, glutamate pyruvate transaminase, and plasma proteins $\mathrm{C}-14$ and D-05). No evidence for questioning parentage as stated was found. We attribute the findings to the occurrence of a deficiency or null variant (cf. Vavrusa et al. 1983). In each instance, the staining intensity of the polypeptide in the presumed carrier of the null was reduced.

$D-05$. In this system, six exceptional mother-father-child combinations were encountered in the Japanese sample. They were of the same type encountered for C-04, and are susceptible to the same explanation. Again there were no parentage exclusions in the families, with the same battery of test systems as previously, but substituting C-04 for D-05 in the battery. We have determined what allele frequencies would account for the observed phenotype proportions, on the assumption of the presence of a silent allele. Because the $V_{2}$ type was only encountered as a heterozygote, the corresponding allele frequency remains the same, and the adjustment is at the expense of $p$ and $q_{v 1}$. The iteration solution of this problem which most closely approximated the observed phenotype frequences was: $\mathrm{p}=0.50, \mathrm{q}=0.41, r=0.03$, and $s$ (silent) $=0.06$. Again, the staining intensity of the polypeptide in question was reduced in the presumed carrier of the null allele.

\section{Discussion}

\section{Nature of variants}

Several general points may be made concerning the nature of the six previously undescribed electrophoretic variants encountered in this study. Five are predominantly in the IEF axis, and one in the molecular weight axis. When these numbers are combined with those available from previous studies under identical conditions on plasma (Rosenblum et al. $1983 \mathrm{a}, \mathrm{b}$ ) and lysate (Rosenblum et al. 1984), we find that about $20 \%$ of our variants are in the molecular weight axis. It is not clear whether variants of this type would be detected with 1-DE. For a similar proportion of the variants described thus far, coordinate variation in two or more polypeptides has

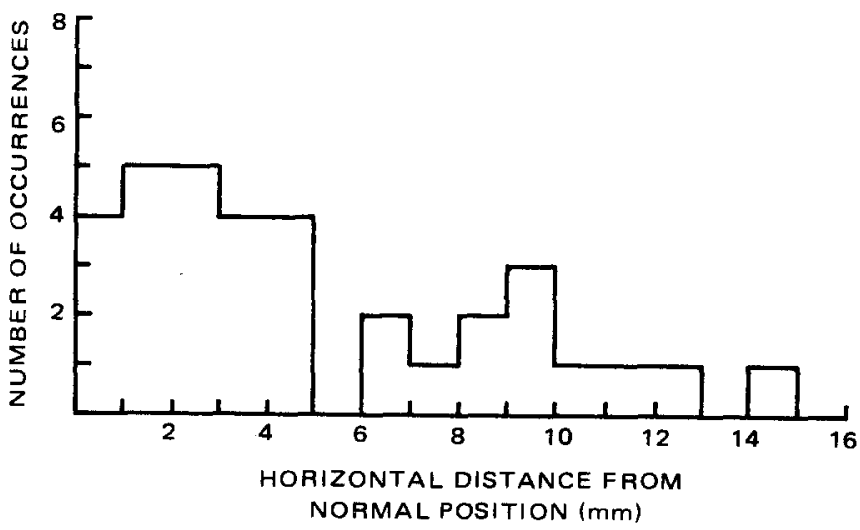

Fig.5. Horizontal gel distance separating the 34 variant polypeptides encountered in our various studies from the normal position. Further explanation in text

been observed, the exact fraction depending on how such variation is defined. The reasons for this coordinate variation are undoubtedly as diverse as the various explanations for multiple bands in isozyme patterns.

With respect to the 34 different variants involving primarily shifts in the IEF axis encountered in our six technically similar studies (Rosenblum et al. 1983a,b, 1984; Baier et al. 1984; this manuscript; Hanash et al. to be published), we have plotted in Fig. 5 the distance between the position assumed by the variant and the presumed normal position. Earlier we have demonstrated that in the case of relatively small polypeptides known to differ from the normal by a gain or loss of one or two molecular charges, such as several of the $\beta$-chain hemoglobin or carbonic anhydrase variants, in a modification of this system, a one-charge change induced a shift of $5-7 \mathrm{~mm}$ in the IEF axis (Wanner et al. 1982). The distribution of Fig. 5 departs significantly from the Poisson $\left(\chi^{2}=254, d f=13\right.$, $P<0.001$ ). It is tempting to see in this distribution a bimodality, with one outlier to the right, and a curtailed distribution to the left. It remains for the future to determine the precise kinds of genetic events that contribute to this unusual distribution. But with all due caution, given that most of the variants we have thus far encountered involve larger polypeptides than those with which the effect of a charge-change on mobility was standardized, we suggest that the first mode is predominantly the result of variants resulting from amino acid substitutions characterized by one-charge changes (plus some "neutral" amino acid substitutions which alter molecular configuration even under these denaturing conditions). Amino acid substitutions resulting in two-charge changes would then be an important contributor to the second mode.

\section{Heterozygosity indices}

Rosenblum et al. (1983b) found a heterozygosity index of $6.2 \pm 0.7 \%$ for the 20 "randomly selected" silver stained polypeptides which they scored for genetic variation in Caucasoids. Only 11 of these polypeptides could be routinely scored in Amerindians. If we limit the interethic comparisons of heterozygosity to these 11 polypeptides, the resulting heterozygosity indices are as follows: Amerindians, $4.5 \pm 0.6 \%$; Japanese, $5.7 \pm 0.7 \%$; and Caucasians, $8.0 \pm 1.1 \%$. For the 12 polypeptides scored after Coomassie Blue staining, not in any sense a random sample, the results were: Amerindians, $3.2 \pm 0.5 \%$; Japanese, $4.0 \pm 0.5 \%$; and Caucasians, $8.6 \pm 1.1 \%$. We have 
previously reported that for a series of 28 erythrocyte enzymes and serum proteins scored for genetic variability by 1-DE in Amerindians, Japanese, and Caucasians, the determinations all performed with comparable techniques, the indices of heterozygosity were $5.4 \%, 7.7 \%$, and $7.8 \%$, respectively (Neel 1978). The present results confirm the somewhat higher index of the Caucasoids, and lower of the Amerindians, but now the Japanese are more similar to the Amerindians than previously. These results also confirm in general our previous finding with the 2-D PAGE technique, of more genetic variability in plasma proteins than reported by other investigators for different types of preparations. As previously noted, we have also reported heterozygosity indices, using the 2-D PAGE technique, of $3.1 \pm 0.5 \%$ in "randomly selected" polypeptides of erythrocyte lysates (Rosenblum et al. 1984). Although we have yet to examine the precise materials studied by the earlier investigators, we are increasingly inclined to suspect that our demonstration of greater amounts of genetic variation rests as much on technical factors as on differences in the nature of the material studied.

\section{Ethnic differences with respect to variants of specific polypeptides}

In addition to the emerging differences in heterozygosity indices, the present data point to a number of other potential differences of anthropologic and genetic interest. The numbers are still relatively small, and all conclusions are tentative. In this spirit, we draw attention to the absence from both these groups of a variant of $\mathrm{C}-01$ encountered in polymorphic frequencies in Caucasoids but the presence in both groups of a variant of C-11 (apolipoprotein A-IV) different from the variant commonly encountered in Caucasoids. For A-02 and D-05, the Japanese possess in polymorphic proportions variants not detected in either of the other two groups. For B-03 there appear to be major differences in the frequency of a variant allele between Caucasoids and the two mongoloid groups. Finally, for C-03 ( $\alpha_{1}$-antitrypsin), the data appear to confirm the known higher frequency of variants of this protein in Caucasoids than Japanese (Ashley et al. 1980).

\section{2-D PAGE: The broader context}

It is apparent from the emerging ethnic differences-which we propose shortly to study further with examinations of 2-D PAGE preparations of erythrocyte lysates, platelets, and lymphocytes-that an important new tool in the study of evolutionary relationships is at hand. Furthermore, we expect, now that the usefulness of 1-DE in the study of mutation has been demonstrated (in Neel 1983), that the 2-D PAGE approach will become "second generation" technology in that context; studies such as the present are part of the necessary familiarizations. Finally, given the number of exon products which can be evaluated with 2-D PAGE, it will in time be of great interest to contrast the results of studies such as this with the data becoming available on nucleotide substitutions in exons as determined by the direct approach.

Acknowledgements. This study was supported through a contract of the U.S. Department of Energy with the University of Michigan, as well as by the Radiation Effects Research Foundation, a private, nonprofit Japanese Foundation supported equally by the Government of Japan through the Ministry of Health and Welfare and the Govern- ment of the United States through the National Academy of Sciences under contract with the Department of Energy. We thank Dr. S. Koga of Kyushu University School of Medicine for a sample of antiserum against human apolipoprotein A-IV.

\section{References}

Anderson L, Anderson NG (1977) High resolution two-dimensional electrophoresis of human plasma proteins. Proc Natl Acad Sci USA 74:5421-5425

Asakawa J, Takahashi N, Rosenblum BB, Neel JV (1984) Analysis of genetic variation in Amerindian sera by 2-D PAGE. In: Hirai $H$ (ed) Electrophoresis '83. de Gruyter, Berlin New York, pp 189194

Ashley MJ, Chan-Yeung M, Corey PN (1980) $P_{i}$ phenotypes in North American workers: Racial differences and comparisons with reported frequencies. Hum Hered 30:107-111

Baier LJ, Hanash SM, Erickson RP (1984) Mouse liver protein variants detected by two-dimensional electrophoresis. In: Hirai $\mathrm{H}$ (ed) Electrophoresis '83. de Gruyter, Berlin New York, pp 189194

Barrantes R, Smouse PE, Neel JV, Mohrenweiser HW, Gershowitz $H$ (1982) Migration and genetic infrastructure of the Central American Guaymi and their affinities with other tribal groups. Am J Phys Anthropol 58:201-214

Børresen A-L, Berg K (1981) The apoE polymorphism studied by two-dimensional high-resolution gel electrophoresis of serum. Clin Genet $20: 438-448$

Brown WT (1982) $\alpha_{1}$-Antitrypsin: Apparent molecular weight heterogeneity shown by two-dimensional electrophoresis. Am J Hum Genet $34: 195-208$

Comings DE (1982) Two-dimensional gel electrophoresis of human brain proteins. III. Genetic and non-genetic variations in 145 brains. Clin Chem 28:798-804

Goldman D, Merril CR (1983) Human lymphocyte polymorphisms detected by quantitative two-dimensional electrophoresis. Am J Hum Genet $35: 827-837$

Hamaguchi H, Ohta A, Mukai R, Yabe T, Yamada M (1981) Genetic analysis of human lymphocyte proteins by two-dimensional gel electrophoresis. 1. Detection of genetic variant polypeptides in PHA-stimulated peripheral blood lymphocytes. Hum Genet $59: 215-220$

Hamaguchi H, Yamada M, Noguchi A, Fujii K, Shibasaki M, Mukai R, Yabe T, Kondo I (1982a) Genetic analysis of human lymphocyte proteins by two-dimensional gel electrophoresis: 2. Genetic polymorphism of lymphocyte cytosol $64 \mathrm{~K}$ polypeptide. Hum Genet 60:176-180

Hamaguchi H, Yamada M, Shibasaki M, Mukai R, Yabe T, Kondo I (1982b) Genetic analysis of human lymphocyte proteins by twodimensional gel electrophoresis: 3 . Frequent occurrence of genetic variants in some abundant polypeptides of PHA-stimulated peripheral blood lymphocytes. Hum Genet 62:142-147

Hamaguchi H, Yamada M, Shibasaki M, Kondo I (1982c) Genetic analysis of human lymphocyte proteins by two-dimensional gel electrophoresis: 4. Genetic polymorphism of cytosol $100 \mathrm{k}$ polypeptide. Hum Genet $62: 148-151$

Hanash SM, Rosenblum BB, Neel JV, Baier LJ, Markel D, Niezgoda W (1985) Genetic analysis of fifty-two platelet polypeptides detected in two-dimensional polyacrylamide gels. Am J Hum Genet (in press)

Hobart MJ (1979) Genetic polymorphism of human plasminogen. Ann Hum Genet 42: 419-423

Kera Y, Yamasawa K, Kimura S (1983) Phenotypic variation of human antithrombin III in normal plasma: Detection by isoelectric focusing. Jpn J Hum Genet 28:249-253

McConkey EH, Taylor BJ, Phan D (1979) Human heterozygosity: A new estimate. Proc Natl Acad Sci USA 76:6500-6504

Mourant AE, Kopec AC, Domaniewska-Sobczak K (1976) The distribution of the human blood groups and other polymorphisms, 2nd edn. Oxford University Press, London 
Neel JV (1978) Rare variants, private polymorphisms, and locus heterozygosity in Amerindian populations. Am J Hum Genet 30: $465-490$

Neel JV (1983) Frequency of spontaneous and induced "point" mutations in higher eukaryotes. J Hered 74:2-15

Neel JV, Salzano FM, Junqueira PC, Keiter F, Maybury-Lewis D (1964) Studies on the Xavante Indians of the Brazilian Mato Grosso. Am J Hum Genet 16:52-140

Neel JV, Gershowitz H, Mohrenweiser HW, Amos B, Kostyu DD, Salzano FM, Mestriner MA, Lawrence D, Simoes AL, Smouse PE, Oliver WJ, Spielman RS, Neel JV Jr (1980a) Genetic studies on the Ticuna, an enigmatic tribe of Central Amazonas. Ann Hum Genet 44:37-54

Neel JV, Satoh C, Hamilton HB, Otake M, Goriki K, Kageoka T, Fujita M, Neriishi S, Asakawa J (1980b) Search for mutations affecting protein structure in children of atomic bomb survivors: Preliminary report. Proc Natl Acad Sci USA 77:4221-4225

Neel JV, Rosenblum BB, Sing CF, Skolnick MM, Hanash SM, Sternberg S (1984) Adapting two-dimensional gel electrophoresis to the study of human germ-line mutation rates. In: Celis JE, Bravo $\mathrm{R}$ (eds) Methods and applications of two dimensional gel electrophoresis of proteins. Academic Press, New York, pp 259-306

Olaisen B, Teisberg P, Gedde-Dahl T (1982) Fibrinogen $\gamma$ chain locus is on chromosome 4 in man. Hum Genet 61:24-26

Rosenblum BB, Hanash SM, Neel JV (1983a) High-resolution separation of plasma proteins, applications to genetic analysis. In: Galteau M-M, Siest G, Henny J (eds) Biologie Prospective $5^{\circ}$ Colloque International de Pont-a-Mousson. Masson, Paris, pp 85-90

Rosenblum BB, Neel JV, Hanash SM (1983b) Two-dimensional electrophoresis of plasma polypeptides reveals "high" heterozygosity indices. Proc Natl Acad Sci USA 80:5002-5006
Rosenblum BB, Neel JV, Hanash SM, Joseph JL, Yew N (1984) Identification of genetic variants in erythrocyte lysate by twodimensional gel electrophoresis. Am J Hum Genet 36:601-612

Sammons DW, Adams LD, Nishizawa EE (1981) UItrasensitive silver-based color staining of polypeptides in polyacrylamide gels. Electrophoresis 2:135-141

Smith SC, Racine RR, Langley CH (1980) Lack of genic variation in the abundant proteins of human kidney. Genetics 96:967-974

Tracy RP, Currie RM, Young DS (1982) Two-dimensional gel electrophoresis of serum specimens from a normal population. Clin Chem 28:890-899

Utermann G, Steinmetz A, Weber W (1982) Genetic control of human apolipoprotein E polymorphism: Comparison of one- and two-dimensional techniques of isoprotein analysis. Hum Genet 60:344-351

Vavrusa B, Cleve H, Constans $J$ (1983) A deficiency mutant of the Gc system. Hum Genet $65: 102-107$

Walton KE, Styer D, Gruenstein EI (1979) Genetic polymorphism in normal human fibroblasts as analyzed by two-dimensional polyacrylamide gel electrophoresis. J Biol Chem 254:7951-7960

Wanner LA, Neel JV, Meisler MH (1982) Separation of allelic variants by two-dimensional electrophoresis. Am J Hum Genet $34: 209-215$

Zannis VI, Just PW, Breslow JL (1981) Human apolipoprotein E isoprotein subclasses are genetically determined. Am J Hum Genet $33: 11-24$

Received November 15, 1984 / Revised March 18, 1985 